\title{
Efficient use of the velocity gradients tensor in flow modelling
}

\author{
CEES W. PASSCHIER \\ Instituut voor Aardwetenschappen, Rijksuniversiteit Utrecht, Postbus 80.021, 3508 TA Utrecht (The Netherlands)
}

(Received December 9, 1986; accepted January 30, 1987)

\begin{abstract}
Passchier, C.W., 1987. Efficient use of the velocity gradients tensor in flow modelling. Tectonophysics, 136:159-163.

For models of fabric development in rocks, with vorticity as a variable parameter, the choice of an unsuitable reference frame for instantaneous flow can hamper clear presentation of results. The orientation of most fabric elements which develop in deforming rocks is attached to some principal direction of instantaneous flow: syntaxial fibres, crystallographic preferred orientation patterns, steady state foliations and immobilised rigid objects seem to orient themselves in a fixed position relative to instantaneous stretching axes $\left(d_{i}\right)$, while shape fabric elements and mica preferred orientation approach parallelism with the extensional apophysis $\left(l_{1}\right)$ of the flow. Models for development of such fabric elements benefit from a choice of reference frame attached to either $d_{i}$ or $l_{1}$.
\end{abstract}

\section{Introduction}

In modern structural geology, mathematical modelling of the development of fabric elements such as occur in naturally deformed rocks is becoming increasingly important (Ghosh and Ramberg, 1976; McKenzie and Jackson, 1983; Passchier, 1986; Bobyarchick, 1986). The models commonly treat fabric development in a matrix deforming by homogeneous flow, described by the velocity gradients tensor $\boldsymbol{L}$ (Malvern, 1969). Studies which analyse the effect of changing vorticity on fabric development (such as the rotational behaviour of rigid bodies in a ductile medium) can be presented by simple equations and diagrams if the principal directions of $\boldsymbol{L}$ are kept fixed in the external reference frame. This fact is not generally realised and is therefore explained in this paper. The treatment is restricted here to two-dimensional or plane strain flow, but can easily be extended to more general flow types.

\section{Velocity gradients tensor}

In a homogeneously deforming body, the instantaneous velocity of particles at $X$ in a coordinate system $\overline{\boldsymbol{X}}$ can be described by the Eulerian rate of displacement equations:

$\dot{X}_{\mathrm{i}}=L_{i j} X_{j}$

where $\boldsymbol{L}$ is the velocity gradients tensor. $\boldsymbol{L}$ can be decomposed into a symmetric rate of deformation tensor $\boldsymbol{D}$ and antisymmetric vorticity tensor $\boldsymbol{W}$ according to Malvern (1969, p. 146) and Means et al. (1980):

$L_{i j}=D_{i j}+W_{i j}$

The column vectors of $\boldsymbol{L}$ can be used to construct a Mohr circle of the first kind (De Paor and Means, 1984) representing the tensor. This is done using a convention first given by Means (1983) to plot the angular velocity $(\omega)$ and stretching rate $(\dot{\epsilon})$ of material lines instantaneously coinciding with the axes of the coordinate system as points in 
$\dot{\epsilon}-\omega$ space following:

$L_{11},-L_{21}=(\dot{\epsilon}, \omega)^{X_{1}}$

$L_{22}, L_{12}=(\dot{\epsilon}, \omega)^{X_{2}}$

An off-axis Mohr circle can now be drawn through both points and centred on the connecting line (Fig. 1; cf. Lister and Williams, 1983; Means, 1983). Each point on the Mohr circle represents $\dot{\epsilon}$ and $\omega$ values of a material line and the angle between two points on the circle is double the angle between material lines in real space (Means, 1983). The coordinates of the centre of the circle are $(V, W / 2)$, representing instantaneous volume change $(V)$ and half the vorticity $(W)$ of the flow. Isochoric flow types plot as circles centred on the $\omega$-axis, coaxial flow types (pure shear) as circles centred on the $\dot{\epsilon}$-axis. In Fig. 1 the diagonally opposite points $\boldsymbol{d}_{1}$ and $\boldsymbol{d}_{2}$ represent the orthogonal eigenvectors of $\boldsymbol{D}$; they are the instantaneous stretching axes of the flow along which maximum $\left(d_{1}\right)$ and minimum $\left(d_{2}\right)$ stretching rates of material lines are realised. Intersections $l_{1}$ and $l_{2}$ of the Mohr circle with the $\dot{\epsilon}$-axis represent eigenvectors of $L$ (Bobyarchick, 1986; Passchier, 1986), sometimes called "flow apophyses" (Ramberg, 1975; Passchier, 1986): they are the lines of no instantaneous angular velocity, which are not usually orthogonal and only exist if eigenvalues of $\boldsymbol{L}$ are real. Non-coaxiality of the flow, i.e. non-coincidence of the centre of the Mohr circle with the $\dot{\epsilon}$-axis, can be expressed by the kinematic vorticity number of Truesdell (1954):

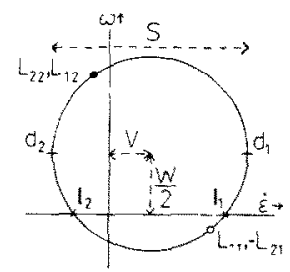

Fig. 1. Mohr circle presentation of velocity gradients tensor $\boldsymbol{L}$. Points on Mohr circle represent instantaneous stretching rate $(c)$ and angular velocity $(\omega)$ of material lines. $W$-vorticity; $V$ - volume change rate; $S$-stretching rate factor, diameter of Mohr circle; $d_{1}, d_{2}$ and $l_{1}, l_{2}$-eigenvectors of $D$ and $L$; open and closed circles-orientation of $X_{1}$ and $X_{2}$ axes of coordinate system.
$W_{\mathrm{k}}=W \cdot\left(2 d_{1}^{2}+2 d_{2}^{2}\right)^{-1 / 2}$

In this paper only isochoric flow is considered, in which case $d_{1}=-d_{2}$ and:

$W_{\mathrm{k}}=W / 2 d_{1}=W / S$

where $S$ is a stretch factor coinciding with the diameter of the Mohr circle (Fig. 1; Lister and Williams, 1983). For simple shear flow $W_{\mathrm{k}}=1$ and for pure shear flow $W_{\mathrm{k}}=0$ (Means et al., 1980).

\section{Reference frames for $L$}

As a tensor, $\boldsymbol{L}$ can be presented in a reference frame of any orientation. Rotation of the reference frame by coordinate transformation causes a change in actual values of matrix coefficients of $\boldsymbol{L}$ and in the position of points representing coordinate axes on the Mohr circle (Fig. 1; Means, 1983) but leaves tensor invariants unmodified. This principle is illustrated in Fig. 2 where three different tensors $\boldsymbol{L}$ are each presented in four orientations by a Mohr circle, in spacial orientation of eigenvectors and in matrix form. Presentation $I$ is a random orientation, II is often used to present simple shear flow (IIc), III for pure shear flow (IIIa) and IV for general non-coaxial flows (IVb), sometimes called sub-simple shear (De Paor, 1983; Bobyarchick, 1986). II and III are related by a $45^{\circ}$ rigid body rotation.

In presentations I, II and III, a change in $W_{\mathrm{k}}$ does not affect the orientation of $d_{1}$ and $d_{2}$ in the external reference frame, but changes the angle $\alpha$ between $l_{1}$ and $l_{2}$ according to:

$\cos \alpha=W_{\mathrm{k}}$

$\sin \alpha=\sqrt{1-W_{\mathrm{k}}^{2}}=2 l_{1} / S$

This is illustrated in $\dot{\epsilon}-\omega$ space by a shift of the Mohr circle along the $\omega$-axis (Figs. 1 and 2).

Presentation IV is rather special in that one of the eigenvectors of $L\left(\boldsymbol{l}_{1}\right.$ in Fig. 2-IV) is permanently fixed in the external reference frame. This implies that $\boldsymbol{d}_{1}$ and $\boldsymbol{d}_{2}$ rotate in the external frame by an angle $\alpha / 2$ upon change of $W_{\mathrm{k}}$. The angle between $d_{1}$ or $d_{2}$ in the first three presentations and in IV is therefore a function of $W_{\mathrm{k}}$ (Fig. 2). 

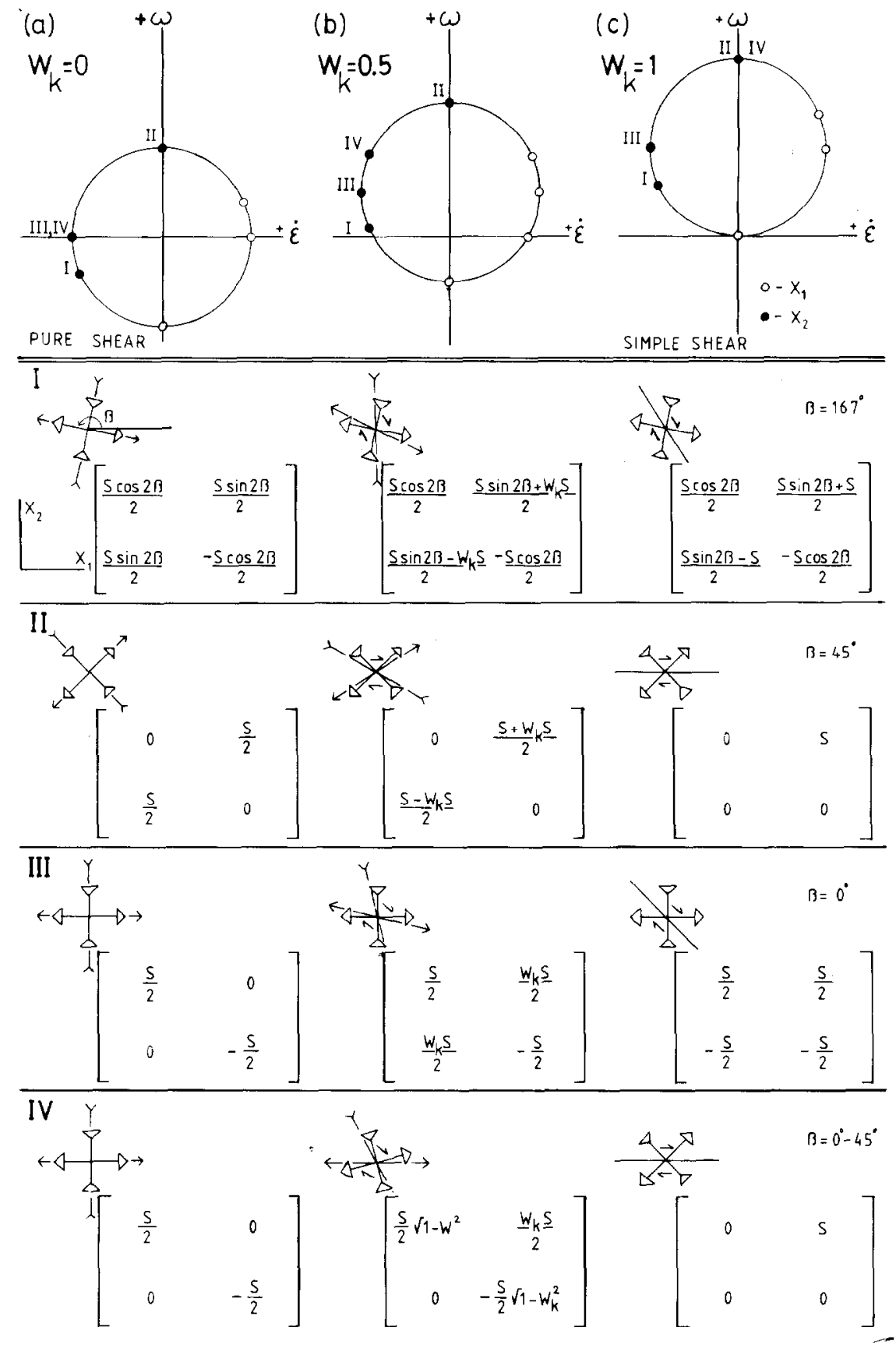

Fig. 2. Presentation of velocity gradients tensor $\boldsymbol{L}$ in Mohr space, real space and in matrix form for three values of $W_{\mathbf{k}}$. Diagrams I-IV show four differently oriented presentations of $\boldsymbol{L}$ in the coordinate system $X_{1}-X_{2} . \beta$-angle between $X_{1}$ and $\boldsymbol{d}_{1}$, measured anticlockwise; $\dot{\epsilon}$ and $\omega$-instantaneous stretching rate and angular velocity of material lines; $W_{\mathrm{k}}-$ kinematic vorticity number; $S$-stretching rate factor, diameter of Mohr circle.

In many recent models of fabric development, the effect of a flow type with variable $W_{\mathrm{k}}$ is studied instead of pure or simple shear only (e.g., Ramberg, 1975; Ghosh and Ramberg, 1976; Mc-
Kenzie and Jackson, 1983; Hanmer, 1984; Freeman, 1985; Bobyarchick, 1986). For this purpose, presentation IV of $\boldsymbol{L}$ is commonly used (ibid.) although it is difficult to see to which advantage. 
In some papers (Ghosh and Ramberg, 1976; Bobyarchick, 1986), the only reason seems to be that a presentation of $\boldsymbol{L}$ with variable $W_{\mathrm{k}}$ can be constructed by addition of pure and simple shear velocity gradients tensors. Since this is done using the standard presentations IIc and IIIa of Fig. 2, which differ by a $45^{\circ}$ rotation of eigenvectors in the external reference frame, a type IV presentation of $\boldsymbol{L}$ results. For many studies, uncritical use of this presentation can unnecessarily complicate the mathematical treatment and hamper clear presentation of results. The rotational behaviour of rigid objects is treated as an example.

\section{Rotating rigid objects}

Ghosh and Ramberg (1976) and Freeman (1985) have investigated the rotational behaviour of ellipsoidal rigid objects in a homogeneously flowing viscous matrix using presentation IV for $\boldsymbol{L}$. Ghosh and Ramberg (1976) find that the angular velocities of object symmetry axes are a function of the object axial ratio, $W_{\mathrm{k}}$ and initial orientation. Equations are given which define the critical positions where symmetry axes have maximum $\left(\boldsymbol{p}_{1}\right)$ and minimum $\left(\boldsymbol{p}_{2}\right)$ angular velocities or become irrotational for some $W_{\mathrm{k}}$ and axial ratio. Careful investigation of these equations shows that the critical positions are symmetrically arranged with respect to $\boldsymbol{d}_{1}$ and $\boldsymbol{d}_{2}$, and remain so upon changes in $W_{\mathrm{k}}$ or axial ratio of the object (Fig. 3; Passchier, in press). $\boldsymbol{p}_{1}$ and $\boldsymbol{p}_{2}$ are orthogonal and fixed at $45^{\circ}$ to $\boldsymbol{d}_{1}$ and $\boldsymbol{d}_{2}$, irrespective of $W_{\mathrm{k}}$ and object axial ratio. These important results are not immediately obvious in the equations and diagrams of Ghosh and Ramberg (1976) due to their use of presentation IV for $\boldsymbol{L}$; all critical positions of object symmetry axes, $\boldsymbol{d}_{1}$ and $\boldsymbol{d}_{2}$ are subject to an extra rotation over $\alpha / 2$ in the external reference frame upon change in $w_{\mathrm{k}}$ (Fig. 3a). The diagrams in Freeman (1985) which demonstrate actual orbits of object symmetry axes for non-plane strain flows, show the same rotation over $\alpha / 2$ for diagrams with different $W_{\mathbf{k}}$. In contrast, Passchier (in press) uses presentation II for $\boldsymbol{L}$ in which $\boldsymbol{d}_{1}$ and $\boldsymbol{d}_{2}$ do not rotate in the external reference frame upon change of $W_{\mathrm{k}}$ (Fig. $3 \mathrm{~b}$ ): this leads to relatively simple equations and diagrams in which

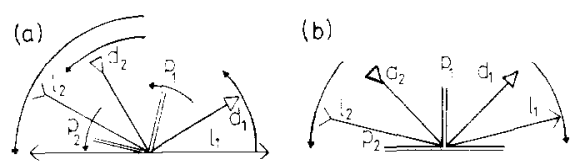

Fig. 3. Change in orientation (curved arrows) of $d_{1}, d_{2}, l_{1}, l_{2}$ and of positions of maximum and minimum angular velocity of rigid objects in the flow $\left(\mathbf{p}_{1}, \mathbf{p}_{2}\right)$ with $W_{\mathrm{k}}$ increasing from 0 to 1 . a. $l_{1}$ fixed in external reference frame (presentation IV in Fig. 2). b. $d_{1}$ and $d_{2}$ fixed in external reference frame (presentation $I I$ in Fig. 2). Further explanation in text.

the permanently symmetrical arrangement of $\boldsymbol{p}_{1}$, $p_{2}$ and other critical positions with respect to $d_{1}$ and $\boldsymbol{d}_{2}$ is immediately obvious.

\section{Orientation of fabric elements}

An obvious question arising from the foregoing is whether presentation IV of $\boldsymbol{L}$ is advantageous in any situation. Many simple fabric elements which develop in response to ductile deformation in rocks seem to have a fixed orientation in the kinematic frame of the flow. Some fabric elements such as syntaxial fibres (Ramsay and Huber, 1983), crystallographic preferred orientation patterns (Lister and Hobbs, 1980), steady-state foliations (Means, 1981) and stable positions of ellipsoidal rigid objects (Ghosh and Ramberg, 1976; Passchier, in press) seem to be fixed to eigenvectors of $D$. They can obviously be modelled most efficiently using presentations I, II or III for $\boldsymbol{L}$.

Others, such as mica preferred orientations, planar and linear shape fabric elements (Ramsay and Huber, 1983), tails of recrystallized material around porphyroclasts (Passchier and Simpson, 1986) and pressure shadows (Malavieille et al., 1982) trend towards parallelism with the extensional eigenvector of $\boldsymbol{L}\left(l_{1}\right.$ in this paper) with progressive deformation. Studies of such fabric elements may benefit from the use of presentation IV.

\section{Conclusions}

From the example given above, it is obvious that some care is needed in the choice of a presentation of $\boldsymbol{L}$ in fabric studies. The method of addition of pure and simple shear tensors to ob- 
tain a general velocity gradients tensor $\boldsymbol{L}$ should be used with care and only for suitable subjects. In other cases, problems can be avoided by use of the method of Means (1983) to derive components of the matrix notation of $\boldsymbol{L}$ directly from a Mohr circle presentation. This method seems most useful because of the visual control over the way in which the components are derived.

\section{References}

Bobyarchick. A.R., 1986. The eigenvalues of steady flow in Mohr space. Tectonophysics, 122: 35-51.

De Paor, D.G., 1983. Orthographic analysis of geological structures. I. Deformation theory. J. Struct. Geol., 5: 255-277.

De Paor, D. and Means, W.D., 1984. Mohr circles of the first and second kind and their use to represent tensor operations. J. Struct. Geol., 6: 693-701.

Freeman, B., 1985. The motion of rigid ellipsoidal particles in slow flows. Tectonophysics, 113: 163-183.

Ghosh, S.K. and Ramberg, H., 1976. Reorientation of inclusions by combination of pure and simple shear. Tectonophysics, 34: 1-70.

Hanmer, S.K., 1984. The potential use of planar and elliptical structures as indicators of strain regime and kinematics of tectonic flow. Current Res., 84: 133-142.

I.ister, G.S. and Hobbs, B.F., 1980. The simulation of fabric development during plastic deformation and its application to quartzite: the influence of deformation history. J. Struct. Geol., 2: 355-371.

Lister, G.S. and Williams, P.F., 1983. The partitioning of deformation in flowing rock masses. Tectonophysics, 92: 1-33.

Malavieille, J., Etchecopar, A. and Burg, J-P., 1982. Analyse de la géométrie des zones abritées: simulation et application à des exemples naturels. C.R. Acad. Sci. Paris, 294: 279-284.

Malvern, L.E., 1969. Introduction to the Mechanics of a continuous medium. Prentice-Hall, Englewood Cliffs, N.J.

McKenzie, D. and Jackson, J., 1983. The relationship between strain rates, crustal thickening, palaeomagnetism, finite strain and fault movements within a deforming zone. Earth Planet. Sci. Lett., 65: 182-202.

Means, W.D., 1981. The concept of steady state foliations. Tectonophysics, 78: 179-199.

Means, W.D., 1983. Application of the Mohr-circle construction to problems of inhomogeneous deformation. J. Struct. Geol., 5: 279-286.

Means, W.D., Hobbs, B.E., Lister, G.S. and Williams, P.F., 1980. Vorticity and non-coaxiality in progressive deformations. J. Struct. Geol., 2: 371-378.

Passchier, C.W., 1986. Flow in natural shear zones-the consequences of spinning flow regimes. Earth Planet. Sci. Lett., 77: 70-80.

Passchier, C.W., in press. Stable positions of rigid objects in non-coaxial flow - a study in vorticity analysis. J. Struct. Geol.

Passchier, C.W. and Simpson, C., 1986. Porphyroclast systems as kinematic indicators. J. Struct. Geol., 8: 831-843.

Ramberg, H., 1975. Particle paths, displacement and progressive strain applicable to rocks. Tectonophysics, 28: 1-37.

Ramsay, J.G. and Huber, M.I., 1983. The Techniques of Modern Structural Geology. Vol. 1. Academic Press, London.

Truesdell, C., 1954. The kinematics of vorticity. Indiana University Press, Bloomington, Ind. 